\title{
Prognostic role of epidermal growth factor receptor in stage III nonsmall cell lung
} cancer

\author{
T. Berghmans*, A.P. Meert*, B. Martin*, V. Ninane" and J.P. Sculier*
}

ABSTRACT: New biological factors have not been extensively studied in stage III nonsmall cell lung cancer (NSCLC). The aim of the present retrospective study was to determine the role of epidermal growth factor receptor (EGF-R) as a prognostic factor in stage III NSCLC, in addition to the stage and other known clinical factors.

Clinical characteristics were retrieved from the patients' charts. Membrane immunostaining for EGF-R was evaluated by three independent observers. The Cox multivariate model, including all variables with a p-value of $<0.2$ in univariate analysis, was used to assess the impact of clinical and biological factors on patients survival.

Between January 1987 and July 2002, 99 assessable stage III NSCLC patients were included in the study. A total of 23 patients were positive for EGF-R (squamous $39.6 \%$ versus nonsquamous 7.8\%). In multivariate analysis, only three factors were statistically significantly associated with survival: performance status, surgery and creatinine.

In conclusion, good performance status, surgical resection and creatinine were found to be independent favourable prognostic factors for survival in a retrospective analysis of stage III nonsmall cell lung cancer, while epidermal growth factor receptor was not even in the univariate analysis.

KEYWORDS: Epidermal growth factor receptor, nonsmall cell lung cancer, prognostic factors, staging

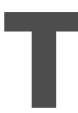
he prognosis of nonsmall cell lung cancer (NSCLC) is generally poor. One of the most important prognostic factors is cancer stage [1]. The last updated version of the International Staging System (ISS) was published in 1997 [2]. This classification needs to be improved, as has been previously suggested by different authors [3-8]. Specifically, for stage III NSCLC, the 1997 ISS separated patients into stages IIIA and IIIB. This group of patients is heterogeneous, including surgically resectable tumours, as well as unresectable diseases treated by chemotherapy and/or radiotherapy. For that reason, some authors have proposed various modifications of the ISS classification [3-8]. In two previous studies $[9,10]$, it was observed that the repartition of stage III NSCLC patients into stages III $\beta$ (tumour/node/metastasis (TNM); T3$4 \mathrm{~N} 3 \mathrm{M} 0$ ) and III $\alpha$ (other TN stage III) better discriminates the patients, in terms of survival, than the 1997 ISS classification.

Some other independent clinical and biological predictors have been identified for predicting survival [11, 12]: age, performance status, serum lactate dehydrogenase (LDH) level, and white blood cell and neutrophil counts. Currently, increased attention has been focused on new biological parameters; biological substaging using molecular markers in a risk stratification strategy has been proposed [13, 14], although their role as prognostic factors in treatment modulation remains unclear. Tumour suppressor genes, proto-oncogenes and markers of metastatic propensity and proliferation are some of the different research tools. Among proto-oncogenes, the epidermal growth factor (EGF) family plays an important role in tumour growth. This phenomenon requires growth regulatory proteins, such as EGF receptor (EGF-R) or protooncogene erbB2. The specific receptor for EGF (EGF-R) is the protein product of the oncogene human EGF-R1. It is a $170-\mathrm{kDa}$ transmembrane glycoprotein (member of the erbB family of cell surface receptors) composed of the following three major regions: an N-terminus extracellular ligand-binding area, a hydrophobic transmembrane domain and a C-terminus intracellular

\section{AFFILIATIONS}

*Dept of Critical Care and Thoracic Oncology, Institut Jules Bordet, and \#Dept of Pneumology, Hôpital SaintPierre, Brussels, Belgium.

CORRESPONDENCE

T. Berghmans

Institut Jules Bordet

Rue Héger-Bordet 1

1000 Bruxelles

Belgium

Fax: 3225343756

E-mail: thierry.berghmans@ bordet.be

Received:

May 212004

Accepted after revision: October 282004 
region with tyrosine kinase activity. The extracellular domain is a ligand-binding site for various polypeptide growth factors: EGF, transforming growth factor- $\alpha$, amphiregulin, betacellulin, heparin-binding protein, epiregulin and vaccinian virus growth factor. The binding of one of these ligands to the extracellular region of EGF-R implies a dimerisation of EGF-R, resulting in autophosphorylation and activation of cytoplasmic signal proteins that are involved in transmitting a mitogenic signal [15]. EGF-R plays a role in cell motility, adhesion, invasion and angiogenesis [16]. Some studies have indicated that the EGF-R signalling pathway may be involved in lung carcinogenesis.

EGF-R is expressed or overexpressed in a wide variety of solid human tumours, including NSCLC, prostate, breast, gastric, colorectal, head and neck, bladder, ovarian cancers and glioblastoma. In NSCLC, EGF-R overexpression is reported in $13-80 \%$ of tumours [17]. Studies have suggested that the expression of high levels of EGF-R is associated with advanced disease and poor prognosis. In a meta-analysis of the published literature, it was found that EGF-R expression, when assessed by immunohistochemistry, was a poor prognostic factor for survival (hazard ratio $(95 \%$ confidence interval (CI)) 1.13 (1.00-1.28)) [17].

As stage III NSCLC is a heterogeneous group, parameters other than disease stage need to be used to improve the choice in therapeutic strategies and prognostic assessment. The aims of the current study were to determine the role of EGF-R as a prognostic factor, specifically in stage III NSCLC, in order to try to improve the 1997 ISS.

\section{MATERIAL AND METHODS Study population}

Since 1985, patients with lung cancer who have been treated in the Dept of Thoracic Oncology at the Institut Jules Bordet, Brussels, Belgium, have been registered in a database. Those presenting with stage III NSCLC, according to the definition of the 1997 ISS [2], untreated before they came to the Dept of Thoracic Oncology, were retrieved from the database and lung biopsies were searched for immunohistochemistry. The last date of inclusion in the study was July 2002, in order to have a minimum follow-up of $1 \mathrm{yr}$ at the time of analysis.

\section{Sample preparation, selection and immunohistochemistry}

All tissues (biopsies or surgical samples $(n=2)$ ) were routinely fixed in $10 \%$ neutral buffered formalin and were embedded in paraffin. From each specimen block, $5-\mu \mathrm{m}$ sections were cut from paraffin-embedded tissues and were deposited on SuperFrost Plus Slides (Menzel-Gläser, Braunschwein, Germany).

All the reagents had an analytical quality and were used without any preliminary purification. Methanol, citric acid, sodium citrate, tris(hydroxymethyl)aminomethane (TRIS) and hydrochloridric acid were purchased from Merck (Darmstad, Germany).

Immunohistochemistry was performed according to a standard avidin-biotin-peroxydase complex, using a mouse monoclonal antibody directed against the external domain of EGF-R (immunoglobulin (Ig)G2a; NCL-EGF-R; clone EGF-R.113;
Novocastra Laboratories, Newcastle Upon Tyne, UK), which produces a membrane staining. Slides were deparaffinised in xylene and rehydrated in ethanol. Endogenous peroxydases were quenched by incubation in $0.3 \%$ hydrogen peroxyde in methanol for $30 \mathrm{~min}$ at room temperature. Slides were rinsed twice in TRIS- $\mathrm{HCl} 0.05 \mathrm{M} \mathrm{NaCl} 0.09 \% \mathrm{pH} 7.6$ for $10 \mathrm{~min}$. They were then submitted to antigen retrieval in citrate buffer $0.01 \mathrm{M} \mathrm{pH} \mathrm{7,} \mathrm{consisting} \mathrm{of} \mathrm{three} \mathrm{5-min} \mathrm{microwave} \mathrm{treatments}$ at $650 \mathrm{~W}$. Slides were recooled for $25 \mathrm{~min}$ at room temperature. Anti-EGF-R antibodies (dilution 1:20; final titration $5 \mu \mathrm{g} \cdot \mathrm{mL}^{-1}$ ) were deposited and incubated for $60 \mathrm{~min}$ at $37^{\circ} \mathrm{C}$. All of the next steps were performed automatically at $37^{\circ} \mathrm{C}$ in the NexES system (Ventana Medical Systems, Tucson, AZ, USA). The complex between EGF-R and its antibodies was fixed with glutaraldehyde $\mathrm{NaCl} 0.9 \%$. The secondary biotinylated antibody was incubated for $8 \mathrm{~min}$. The slides were stained with diaminobenzidine detection kit (Ventana Medical Systems) and counterstained with haematoxylin.

Negative controls for EGF-R were carried out by omitting the primary antibody and also by substituting normal mouse IgG2a with primary antibody. The positive controls were known EGF-R-positive lung adenocarcinomas.

\section{Interpretation of immunohistochemistry}

Three observers (two medical oncologists, T. Berghmans and A.P. Meert, and one biologist, B. Martin) independently evaluated the results of immunohistochemical staining.

The bronchial lesion staining was scored as negative (no immunostaining) or positive (any positive cell with membrane immunostaining) [18]. The results were compared and discordant interpretations were resolved by review of the specific slides by the three observers at a multihead microscope (Olympus BX41; Olympus, Tokyo, Japan).

\section{Statistics}

Survival was measured from the date of diagnosis until the date of death, January 30, 2004 or the last date known alive if earlier. Univariate survival comparisons were performed with a log rank test. All variables with a p-value $\leqslant 0.2$ in univariate analysis were included in a Cox model for multivariable analysis. Comparison of patients' characteristics according to EGF-R status was made by the Chi-squared test, in case of a binary variable, or Mann-Whitney U-test. A p-value of $<0.05$ was considered as statistically significant.

The following variables were used in survival analyses: 1997 ISS stage IIIA/IIIB; the European Lung Cancer Working Party staging system III $\alpha /$ III $\beta$ (other TN stage III/T3-4N3M0); T and $\mathrm{N}$ status; performance status; age; sex; weight loss; histology; white blood cell, neutrophil and platelet counts; haemoglobin level; LDH and alkaline phosphatase levels; surgical intervention; creatinine, calcium, sodium and bilirubin levels; and EGF$\mathrm{R}$ status.

\section{RESULTS}

From January 1987 until July 2002, 298 consecutive patients with stage III NSCLC were treated in the Dept of Thoracic Oncology, Institut Jules Bordet. No biopsies were available for 175 patients, mainly because biopsies were taken in another institution and were not available for immunohistochemistry. 
In 17 cases, there was no more tumoural tissue in the block. Seven patients were excluded because they were not treated, or they had received treatment for their NSCLC in another institution before they were seen in the current authors' hospital. Consequently, 99 stage III NSCLC patients, untreated at the time they went to the current authors' department, were assessable and included in this study. The principal characteristics of the patients are described in table 1. Repartition of the patients according to $\mathrm{T}$ and $\mathrm{N}$ status is reported in table 2.

The majority of the patients were treated either with chemotherapy alone $(n=40)$ or with a combination $(n=42)$, including chemotherapy plus radiotherapy $(n=36)$ or plus surgery with or without radiotherapy $(n=6)$. The other patients received radiotherapy alone $(n=15)$ or were offered surgery only $(n=2)$. Cisplatin or carboplatin-based chemotherapy was administered in 71 and five patients, respectively. Other patients received nonplatinum-containing chemotherapy $(n=6)$, mainly due to functional reasons that contraindicated platinum administration. The response rate to induction therapy was $39.4 \%$.

\section{TABLE 1} Characteristics of 99 patients with stage III nonsmall cell lung cancer (NSCLC)

\section{Characteristics}

\begin{tabular}{lc} 
Age yrs & $64(37-83)$ \\
Males/females & $75 / 24$ \\
Smoking never/active/ex-smoker & $2 / 60 / 36$ \\
Histology & \\
$\quad$ Squamous & 48 \\
Adenocarcinoma & 29 \\
Other NSCLC histology & 22 \\
Weight loss \% & $0(0-25)$ \\
Stage IIIA/IIIB & $40 / 59$ \\
Stage III $\alpha /$ III $\beta$ & $80 / 15$ \\
Karnofsky performance status & $80(20-100)$ \\
Mediastinoscopy yes positive/yes negative/no & $22 / 8 / 69$ \\
Pleural or pericardial effusion & 12 \\
\hline
\end{tabular}

Data are presented as median (range) and $\mathrm{n}$.

TABLE 2 Repartition of tumour (T) and node (N) status in 99 patients with stage III NSCLC

\begin{tabular}{lcccccc} 
& T1 & T2 & T3 & T4 & Tx & Tis \\
\hline N0 & & & & 4 & & \\
N1 & & & 1 & 2 & & \\
N2 & 5 & 25 & 9 & 22 & & \\
N3 & 4 & 7 & 4 & 11 & 3 & 1 \\
Nx & & & & 1 & & \\
\hline
\end{tabular}

Tx: T status cannot be assessed; Tis: in situ carcinoma; Nx: N status cannot be assessed.
A total of 76 and 23 patients, respectively, were negative or positive for EGF-R staining. Overall, the positivity rate $(95 \%$ CI) was $23.2 \%(14.9-31.5)$. It was $39.6 \%$ (19 out of 48 ) for squamous cell carcinoma and $7.8 \%$ (4 out of 51 ) among other histological subtypes $(p=0.0002)$. The characteristics of the patients according to EGF-R status are described in table 3. Except for histology, there was no statistically significant difference between EGF-R positive and negative tumours.

The median follow-up duration was 78.4 months (range 0.8204 months). At the time of analysis, 10 patients were still alive, 83 were dead and six were lost to follow-up. The 1-, 2-, 3- and 4 -yr survival rates were $51.6 \%, 19.8 \%, 9.1 \%$ and $2.4 \%$, respectively. The results of univariate survival analyses are reported in table 4 . The following variables were found to be statistically significantly associated with difference in survival: good performance status, surgical intervention, normal haemoglobin level, normal sodium and $\mathrm{T}$ status. In addition to these five variables, those with a $p$-value $\leqslant 0.2$ in univariate analysis were included in a multivariable Cox model. These were as follows: weight loss, neutrophil and platelet counts, LDH and creatinine. A total of 83 observations were complete. Three variables were independently associated with survival in multivariate analysis: good performance status $(p=0.006)$, the undertaking of surgery $(p=0.007)$ and creatinine level $(p=0.02$; table 5). As there were nine missing values for weight loss, a second multivariate analysis was performed, not including this variable. In total, 91 observations were complete. The same three independent variables were found to be associated with survival.

The same analysis was performed in EGF-R positive and negative tumours separately. For EGF-R positive tumours

\begin{tabular}{lccc} 
TABLE 3 & $\begin{array}{c}\text { Patients' characteristics according to epidermal } \\
\text { growth factor receptor (EGF-R) status }\end{array}$ \\
& EGF-R positive & EGF-R negative & p-value \\
\hline & & & \\
Overall & 23 & 76 & \\
Stage IIIA/IIIB & $9 / 14$ & $31 / 45$ & 0.89 \\
Stage III//III & $19 / 3$ & $61 / 12$ & 0.75 \\
Performance status ${ }^{\#}$ & & & \\
$\quad 80-100$ & 15 & 39 & 0.30 \\
$\quad 60-70$ & 6 & 27 & \\
$\quad<60$ & 1 & 6 & \\
Age yrs & & & \\
$\quad \leqslant 60$ & 7 & 25 & 0.83 \\
$\quad>60$ & 16 & 51 & \\
Male/female & $18 / 5$ & $57 / 19$ & 0.75 \\
Histology & & & \\
$\quad$ Squamous & 19 & 29 & 0.0002 \\
$\quad$ Nonsquamous & 4 & 47 & \\
Weight loss & & & \\
$\quad \leqslant 5 \%$ & 15 & 51 & 0.82 \\
$>5 \%$ & 6 & 18 & \\
\hline
\end{tabular}

Data are presented as $\mathrm{n}$, unless otherwise stated. ${ }^{\#}$ : according to Karnofsky scale. 


\begin{tabular}{|c|c|c|c|c|}
\hline & MST days & Patients & Deaths & $p$-value \\
\hline Overall & 383 & 99 & 83 & \\
\hline EGF-R negative & 371 & 76 & 65 & 0.26 \\
\hline EGF-R positive & 411 & 23 & 18 & \\
\hline IIIA & 408 & 40 & 35 & 0.36 \\
\hline IIIB & 332 & 59 & 48 & \\
\hline IIla & 413 & 80 & 67 & 0.25 \\
\hline III/ & 140 & 15 & 13 & \\
\hline T1-T2 & 448 & 41 & 32 & 0.05 \\
\hline T3-T4 & 263 & 54 & 48 & \\
\hline NO-2 & 413 & 68 & 59 & 0.41 \\
\hline N3 & 215 & 30 & 23 & \\
\hline \multicolumn{5}{|c|}{ Performance status $\#$} \\
\hline 80-100 & 500 & 54 & 43 & 0.001 \\
\hline$<80$ & 220 & 40 & 36 & \\
\hline \multicolumn{5}{|l|}{ Age yrs } \\
\hline$\leqslant 60$ & 424 & 32 & 28 & 0.57 \\
\hline$>60$ & 375 & 67 & 55 & \\
\hline Males & 400 & 75 & 62 & 0.37 \\
\hline Females & 310 & 24 & 21 & \\
\hline \multicolumn{5}{|l|}{ Weight loss } \\
\hline$\leqslant 5 \%$ & 416 & 66 & 55 & 0.13 \\
\hline$>5 \%$ & 219 & 24 & 21 & \\
\hline \multicolumn{5}{|l|}{ Histology } \\
\hline Squamous & 420 & 48 & 41 & 0.46 \\
\hline Nonsquamous & 332 & 51 & 42 & \\
\hline \multicolumn{5}{|l|}{ Surgery } \\
\hline Yes & 1085 & 8 & 4 & 0.00002 \\
\hline No & 365 & 91 & 79 & \\
\hline \multicolumn{5}{|l|}{ WBC } \\
\hline$\leqslant 10^{4} \cdot \mathrm{mm}^{-3}$ & 370 & 64 & 54 & 0.73 \\
\hline$>10^{4} \cdot \mathrm{mm}^{-3}$ & 437 & 35 & 29 & \\
\hline \multicolumn{5}{|l|}{ PMN } \\
\hline$\leqslant 7500 \cdot \mathrm{mm}^{-3}$ & 403 & 67 & 55 & 0.15 \\
\hline$>7500 \cdot \mathrm{mm}^{-3}$ & 332 & 32 & 28 & \\
\hline \multicolumn{5}{|l|}{ Platelets } \\
\hline$\leqslant 44 \times 10^{4} \cdot \mathrm{mm}^{-3}$ & 401 & 78 & 64 & 0.07 \\
\hline$>44 \times 10^{4} \cdot \mathrm{mm}^{-3}$ & 199 & 21 & 19 & \\
\hline \multicolumn{5}{|c|}{ Haemoglobin $\mathbf{g} \cdot \mathrm{dL}^{-1}$} \\
\hline $12-18$ & 401 & 71 & 59 & 0.03 \\
\hline$<12$ & 216 & 28 & 24 & \\
\hline \multicolumn{5}{|l|}{$\mathrm{LDH} \mathrm{mUI} \cdot \mathrm{mL}^{-1}$} \\
\hline$\leqslant 200$ & 419 & 67 & 53 & 0.07 \\
\hline$>200$ & 311 & 31 & 29 & \\
\hline \multicolumn{5}{|l|}{ Creatinine $\mathrm{mg} \cdot \mathrm{dL}^{-1}$} \\
\hline$\leqslant 0.9$ & 412 & 70 & 55 & 0.07 \\
\hline$>0.9$ & 288 & 29 & 28 & \\
\hline \multicolumn{5}{|l|}{ Calcium $\mathrm{mg} \cdot \mathrm{dL}^{-1}$} \\
\hline $8.5-10.3$ & 383 & 89 & 73 & 0.31 \\
\hline$<8.5$ or $>10.3$ & 370 & 9 & 9 & \\
\hline \multicolumn{5}{|l|}{ AP $\mathrm{mUI} \cdot \mathrm{mL}^{-1}$} \\
\hline$\leqslant 110$ & 406 & 57 & 48 & 0.57 \\
\hline$>110$ & 316 & 42 & 35 & \\
\hline \multicolumn{5}{|l|}{ Sodium mEq. $L^{-1}$} \\
\hline $135-146$ & 404 & 83 & 68 & 0.01 \\
\hline$<135$ or $>146$ & 147 & 16 & 15 & \\
\hline
\end{tabular}

\begin{tabular}{lllll} 
TABLE 4 & (Continued) & & & \\
& MST days & Patients & Deaths & p-value \\
\hline $\begin{array}{l}\text { Bilirubin } \mathbf{~ m g} \cdot \mathbf{d L}^{-1} \\
\quad 30.5 \\
>0.5\end{array}$ & 381 & 64 & 55 & 0.59 \\
\hline
\end{tabular}

Data are presented as $n$, unless otherwise stated. MST: median survival time; EGF-R: epidermal growth factor receptor; T: tumour; N: node; WBC: white blood cell count; PMN: polymorphonuclear neutrophil count; LDH: lactate dehydrogenase; AP: alkaline phosphatases. \#: according to Karnofsky scale.

$(n=23)$, only the undertaking of surgery was associated with a survival advantage in univariate analysis $(\mathrm{p}=0.01)$. No independent variable was found in multivariate analysis. In EGF-R negative tumours $(n=76)$, good performance status $(p=0.004)$, the undertaking of surgery $(p=0.0002)$, low creatinine level $(p=0.04)$, normal sodium level $(p=0.02)$ and T1-T2 status $(p=0.01)$ were associated with a survival benefit in univariate analysis. All variables with a $p$-value $\leqslant 0.2$ in univariate analysis were entered in a Cox model. In addition to the previous five variables, 1997 ISS stage IIIA/IIIB $(\mathrm{p}=0.13)$, weight loss $(p=0.07)$, neutrophil count $(p=0.12), \mathrm{LDH}$ $(p=0.13)$, platelet count $(p=0.10)$ and haemoglobin level $(p=0.12)$ were included. Two multivariate analyses were performed. In the first, including stage IIIA/IIIB but not $\mathrm{T}$ status, the undertaking of surgery $(p=0.02)$ and performance status $(p=0.03)$ were significantly associated with survival. In the second model, including $\mathrm{T}$ status but not stage IIIA/IIIB, the undertaking of surgery $(p=0.04)$ and creatinine level $(p=0.05)$ were independently associated with survival.

\section{DISCUSSION}

In this retrospective study assessing the potential prognostic role of EGF-R in stage III NSCLC, it was found that good

TABLE 5 Multivariate analysis for survival in stage III nonsmall cell lung cancer patients $\#$

\begin{tabular}{lccc} 
& $\boldsymbol{\beta}$-Coefficient & Standard error & $\boldsymbol{p}$-value \\
\hline Performance status & 0.88 & 0.32 & 0.006 \\
Surgical resection & 1.73 & 0.64 & 0.007 \\
Creatinine & 0.69 & 0.28 & 0.02 \\
LDH & 0.53 & 0.30 & 0.07 \\
Sodium level & 0.46 & 0.48 & 0.34 \\
Haemoglobin level & 0.27 & 0.33 & 0.40 \\
Platelet count & -0.37 & 0.45 & 0.41 \\
Neutrophil count & 0.26 & 0.33 & 0.44 \\
T status & 0.21 & 0.28 & 0.45 \\
Weight loss & 0.03 & 0.34 & 0.93 \\
\hline
\end{tabular}

Multivariate analysis was performed by the Chi-squared test $\left(\chi^{2}=38.33\right.$, degree of freedom $=10, p=0.00003$ ). LDH: lactate dehydrogenase; $T$ : tumour. $\#: n=83$. 
performance status, the undertaking of surgery and low creatinine level were all associated with better survival. EGF$\mathrm{R}$ did not appear as a prognostic factor for survival in this group of patients.

Stage III NSCLC corresponds to a heterogeneous group of patients that require multimodal therapy, including surgery in some cases, but, generally, chemotherapy and/or radiotherapy. The current ISS classification [2] remains unsatisfactory in terms of the prognosis in patients who are, for the majority, clinically staged. In previous studies, it has been observed that improvement of this staging system can be obtained by using simple clinical parameters [3-9, 19]. In addition to well-known clinical and biological prognostic factors [1], the role of new biological factors with potential prognostic value and, possibly, therapeutic implication was assessed in lung cancer. Among other things, the members of the EGF family, EGF-R and erbB2-neu were evaluated in different studies with frequently conflicting or inconclusive results, such that meta-analyses were required to allow meaningful conclusions. The current authors decided to evaluate the prognostic role of EGF-R in patients with stage III NSCLC for three main reasons. First, to the best of the current authors' knowledge, no study dealing with EGF-R within this group of patients has been published. Secondly, since it was observed in a previous meta-analysis [17] that EGF-R was a poor prognostic factor for survival in NSCLC for all stages (I-IV), at least when assessed by immunohistochemistry, the current authors tried to determine if EGF-R has a prognostic value for survival within stage III disease. Thirdly, the current authors wanted to determine EGF-R positivity rate in stage III NSCLC because EGF-R is a potential therapeutic target for EGF-R tyrosine kinase inhibitors (gefinitib, erlonitib, etc.) or for monoclonal antibodies (cetuximab, etc.). These drugs and others are currently in clinical investigations as molecular "targeted" therapies. Trials with gefinitib in advanced [20, 21] or recurrent NSCLC [22] were recently published. It must be pointed out that EGF-R expression, at least for gefinitib, is not directly related to the treatment response [23]. EGF-R itself is not the only characteristic for the translation pathway of EGF-R. Heterodimerisation of EGF-R with other tyrosine-kinase receptors, principally HER2 [24], or Akt phosphorylation are other important pathways involved in predicting survival [25]. Furthermore, a good correlation was recently demonstrated between EGF-R mutation and response to gefinitib in NSCLC [26, 27]. Nevertheless, it seems more important for monoclonal antibodies to bind to the extracellular domain of EGF-R, for which the presence can be assessed by immunohistochemistry.

No difference was observed in terms of survival between patients with EGF-R positive or negative tumours. Outside of potential biases, which are discussed below, it is possible that the biological behaviour in stage III NSCLC is too homogeneous to bring to the fore any survival difference according to EGF-R status. Moreover, the negative survival impact of EGF-R observed in the meta-analysis was weak [17]. Therefore, a large number of patients are needed to reproduce these results, and the number of patients included in the current study was probably too small to allow it.
Some potential biases could have interfered with the present results. This was a retrospective study, as with the majority of biological studies. Although all consecutive patients treated in the current authors' department were included in a database, not all of the patients were included because there was not enough tumour material in two-thirds of the cases, as expected. Few patients were lost to follow-up (6\%) and they were equally distributed between the EGF-R positive and negative groups. The inclusion period was long, as in other biological studies, but the therapeutic approach remained similar, with chemotherapeutic and radiotherapeutic schedules being fairly equivalent with respect to time. Treatment strategies were based on the protocols that were used at the current authors' institution during the study period [28, 29]. Old chemotherapeutic agents that were considered ineffective were not used, and, except in the case of contraindication, all patients received cisplatin-based chemotherapy, which remains the standard of care for NSCLC chemotherapy.

Another potential source of confusion is related to immunohistochemistry. There is no validation or standardisation of immunohistochemistry techniques to assess EGF-R. An immunohistochemistry protocol was used, which was previously applied by the current authors' group in other studies $[18,30]$. Since no international consensus exists on the cut-off defining tumoural EGF-R positivity, a cut-off that the current authors validated in a previous study was used [18]. The positivity rate that was found in the present study is relatively low, but remained in the range observed in the literature [17]. Nevertheless, a direct comparison among studies assessing EGF-R in NSCLC is difficult because the authors did not use the same cut-off defining EGF-R positivity. Furthermore, cytoplasmic staining was considered by some authors as indicative, although others determined that only membrane staining was positive [17]. These observations emphasise the need for a standardisation of immunohistochemistry procedures that allow powerful comparisons between studies. There could be a risk of bias due to the size of the samples used in the current study. For the majority, small biopsies were used, as compared with most studies where large surgical samples were available. In a previous study, the results of EGF-R immunostaining on biopsy and surgical samples were compared and a good correlation was found [18].

Stage III nonsmall cell lung cancer comprises a heterogeneous group of patients, for which the 1997 International Staging System remains unsatisfactory. Improvements of the International Staging System have been proposed, some of which have potential therapeutic implications, but none include new biological factors. Unfortunately, in the present study, epidermal growth factor receptor did not appear to add any prognostic information to known clinical prognostic factors, such as performance status, in this group of patients with stage III nonsmall cell lung cancer. New studies dealing with other biological factors are underway, including the evaluation of apoptosis, angiogenesis and the metastatic process, with the hope that this will increase the discrimination power of the staging system and, ultimately, allow a better definition of the therapeutic strategy. In conclusion, good performance status, the undertaking of surgery and low creatinine level are the only significant independent prognostic factors in the 
currently studied population of stage III nonsmall cell lung cancer patients.

\section{REFERENCES}

1 Graziano SL. Non-small cell lung cancer: clinical value of new biological predictors. Lung Cancer 1997; 17: Suppl. 1, S37-S58.

2 Mountain CF. Revisions in the International System for Staging Lung Cancer. Chest 1997; 111: 1710-1717.

3 Jassem J, Skokowski J, Dziadziuszko R, et al. Results of surgical treatment of non-small cell lung cancer: validation of the new postoperative pathologic TNM classification. J Thorac Cardiovasc Surg 2000; 119: 1141-1146.

4 Andre F, Grunenwald D, Pignon JP, et al. Survival of patients with resected N2 non-small-cell lung cancer: evidence for a subclassification and implications. J Clin Oncol 2000; 18: 2981-2989.

5 Bollen EC, Theunissen PH, van Duin CJ, Drenth BM, van Noord JA, Blijham GH. Clinical significance of intranodal and extranodal growth in lymph node metastases of nonsmall cell lung cancer. Scand J Thorac Cardiovasc Surg 1994; 28: 97-102.

6 Vansteenkiste JF, De Leyn PR, Deneffe GJ, et al. Survival and prognostic factors in resected N2 non-small cell lung cancer: a study of 140 cases. Leuven Lung Cancer Group. Ann Thorac Surg 1997; 63: 1441-1450.

7 Inoue K, Sato M, Fujimura S, et al. Prognostic assessment of 1310 patients with non-small-cell lung cancer who underwent complete resection from 1980 to 1993. J Thorac Cardiovasc Surg 1998; 116: 407-411.

8 Leong SS, Rocha Lima CM, Sherman CA, Green MR. The 1997 International Staging System for non-small cell lung cancer: have all the issues been addressed? Chest 1999; 115: 242-248.

9 Berghmans T, Lafitte JJ, Thiriaux J, et al. Survival is better predicted with a new classification of stage III unresectable non-small cell lung carcinoma treated by chemotherapy and radiotherapy. Lung Cancer 2004; 45: 339348.

10 Sculier JP, Paesmans M, Ninane V, et al. Evaluation of the TN sub-staging in patients with initially unresectable stage III non-small cell lung cancer treated by induction chemotherapy. The European Lung Cancer Working Party. Lung Cancer 1998; 22: 201-213.

11 Kanters SD, Lammers JW, Voest EE. Molecular and biological factors in the prognosis of non-small cell lung cancer. Eur Respir J 1995; 8: 1389-1397.

12 Sculier JP, Paesmans M, Libert P, et al. Facteurs pronostiques des cancers bronchiques non à petites cellules au stade avancé: expérience de l'European Lung Cancer Working Party [Prognostic factors in advanced stage non-small cell bronchial cancer: experiences of the European Lung Cancer Working Party]. Rev Mal Respir 1997; 14: 445-449.

13 D'Amico TA, Massey M, Herndon JE, Moore MB, Harpole DH Jr. A biologic risk model for stage I lung cancer: immunohistochemical analysis of 408 patients with the use of ten molecular markers. J Thorac Cardiovasc Surg 1999; 117: 736-743.
14 Pastorino U, Andreola S, Tagliabue E, et al. Immunocytochemical markers in stage I lung cancer: relevance to prognosis. J Clin Oncol 1997; 15: 28582865.

15 Prigent SA, Lemoine NR. The type 1 (EGFR-related) family of growth factor receptors and their ligands. Prog Growth Factor Res 1992; 4: 1-24.

16 de Jong JS, van Diest PJ, van der Valk P, Baak JP. Expression of growth factors, growth-inhibiting factors, and their receptors in invasive breast cancer. II: correlations with proliferation and angiogenesis. J Pathol 1998; 184: 53-57.

17 Meert AP, Martin B, Delmotte P, et al. The role of EGF-R expression on patient survival in lung cancer: a systematic review with meta-analysis. Eur Respir J 2002; 20: 975981.

18 Meert AP, Martin B, Verdebout JM, et al. Corrélation entre l'expression d'EGFR dans les biopsies et les pièces chirurgicales des cancers bronchiques [EGF-R expression correlation between biopsy and surgical specimens of lung carcinoma]. Rev Mal Respir 2003; 20: 347-353.

19 Sculier JP, Berghmans T, Castaigne C, et al. Maintenance chemotherapy for small cell lung cancer: a critical review of the literature. Lung Cancer 1998; 19: 141-151.

20 Herbst RS, Giaccone G, Schiller JH, et al. Gefitinib in combination with paclitaxel and carboplatin in advanced non-small-cell lung cancer: a phase III trial INTACT 2. J Clin Oncol 2004; 22: 785-794.

21 Giaccone G, Herbst RS, Manegold C, et al. Gefitinib in combination with gemcitabine and cisplatin in advanced non-small-cell lung cancer: a phase III trial INTACT 1. J Clin Oncol 2004; 22: 777-784.

22 Kris MG, Natale RB, Herbst RS, et al. Efficacy of gefitinib, an inhibitor of the epidermal growth factor receptor tyrosine kinase, in symptomatic patients with non-small cell lung cancer: a randomized trial. JAMA 2003; 290: 2149-2158.

23 Parra HS, Cavina R, Latteri F, et al. Analysis of epidermal growth factor receptor expression as a predictive factor for response to gefitinib ("Iressa", ZD1839) in non-small-cell lung cancer. Br J Cancer 2004; 91: 208-212.

24 Onn A, Correa AM, Gilcrease M, et al. Synchronous overexpression of epidermal growth factor receptor and HER2-neu protein is a predictor of poor outcome in patients with stage I non-small cell lung cancer. Clin Cancer Res 2004; 10: 136-143.

25 Cappuzzo F, Magrini E, Ceresoli GL, et al. Akt phosphorylation and gefitinib efficacy in patients with advanced non-small-cell lung cancer. J Natl Cancer Inst 2004; 96: 1133-1141.

26 Lynch TJ, Bell DW, Sordella R, et al. Activating mutations in the epidermal growth factor receptor underlying responsiveness of non-small-cell lung cancer to gefitinib. N Engl J Med 2004; 350: 2129-2139.

27 Paez JG, Janne PA, Lee JC, et al. EGFR mutations in lung cancer: correlation with clinical response to gefitinib therapy. Science 2004; 304: 1497-1500.

28 Sculier JP, Paesmans M, Lafitte JJ, et al. A randomised phase III trial comparing consolidation treatment with further chemotherapy to chest irradiation in patients with initially unresectable locoregional non-small-cell 
lung cancer responding to induction chemotherapy. European Lung Cancer Working Party. Ann Oncol 1999; 10: 295-303.

29 Sculier JP, Lafitte JJ, Berghmans T, et al. A phase III randomised study comparing two different dose-intensity regimens as induction chemotherapy followed by thoracic irradiation in patients with advanced locoregional nonsmall-cell lung cancer. Ann Oncol 2004; 15: 399-409.

30 Meert AP, Verdebout JM, Martin B, Ninane V, Feoli F, Sculier JP. Epidermal growth factor receptor expression in pre-invasive and early invasive bronchial lesions. Eur Respir J 2003; 21: 611-615. 